\title{
ORIGINAL
}

\section{Effect of therapeutic drug monitoring-based dose optimization of piperacillin/tazobactam on sepsis-related organ dysfunction in patients with sepsis: a randomized controlled trial}

Stefan Hagel ${ }^{1,2^{*}} \mathbb{0}$, Friedhelm Bach ${ }^{3}$, Thorsten Brenner ${ }^{4,5}$, Hendrik Bracht $^{6}$, Alexander Brinkmann $^{7}$, Thorsten Annecke ${ }^{8,9}$, Andreas Hohn ${ }^{8,10}$, Markus Weigand ${ }^{5}$, Guido Michels ${ }^{11}$, Stefan Kluge ${ }^{12}$, Axel Nierhaus ${ }^{12}$, Dominik Jarczak ${ }^{12}$, Christina König ${ }^{12}$, Dirk Weismannn ${ }^{13}$, Otto Frey ${ }^{14}$, Dominic Witzke ${ }^{3}$, Carsten Müller ${ }^{15}$, Michael Bauer ${ }^{16}$, Michael Kiehntopf ${ }^{17}$, Sophie Neugebauer ${ }^{2,17}$, Thomas Lehmann ${ }^{18}$, Jason A. Roberts 19,20,21 and Mathias W. Pletz ${ }^{1,2}$ on behalf of the TARGET Trial Investigators

(c) 2022 The Author(s)

\begin{abstract}
Purpose: Insufficient antimicrobial exposure is associated with worse outcomes in sepsis. We evaluated whether therapeutic drug monitoring (TDM)-guided antibiotic therapy improves outcomes.

Methods: Randomized, multicenter, controlled trial from January 2017 to December 2019. Adult patients $(n=254)$ with sepsis or septic shock were randomly assigned 1:1 to receive continuous infusion of piperacillin/tazobactam with dosing guided by daily TDM of piperacillin or continuous infusion with a fixed dose ( $13.5 \mathrm{~g} / 24 \mathrm{~h}$ if eGFR $\geq 20 \mathrm{~mL} / \mathrm{min}$ ). Target plasma concentration was four times the minimal inhibitory concentration (range $\pm 20 \%$ ) of the underlying pathogen, respectively, of Pseudomonas aeruginosa in empiric situation. Primary outcome was the mean of daily total Sequential Organ Failure Assessment (SOFA) score up to day 10.

Results: Among 249 evaluable patients (66.3 \pm 13.7 years; female, 30.9\%), there was no significant difference in mean SOFA score between patients with TDM (7.9 points; 95\% Cl 7.1-8.7) and without TDM (8.2 points; 95\% Cl 7.5-9.0) $(p=0.39)$. Patients with TDM-guided therapy showed a lower 28 -day mortality $(21.6 \%$ vs. $25.8 \%$, RR $0.8,95 \% \mathrm{Cl} 0.5-1.3$, $p=0.44)$ and a higher rate of clinical (OR 1.9; 95\% Cl 0.5-6.2, $p=0.30$ ) and microbiological cure (OR $2.4 ; 95 \% \mathrm{Cl} 0.7-7.4$, $p=0.12)$, but these differences did not reach statistical significance. Attainment of target concentration was more common in patients with TDM (37.3\% vs. 14.6\%, OR 4.5, Cl 95\%, 2.9-6.9, $p<0.001)$.
\end{abstract}

\footnotetext{
*Correspondence: stefan.hagel@med.uni-jena.de

${ }^{1}$ Institute for Infectious Diseases and Infection Control, Jena University

Hospital-Friedrich Schiller University Jena, Am Klinikum 1, 07747 Jena,

Germany

Full author information is available at the end of the article
}

\section{县


Conclusion: TDM-guided therapy showed no beneficial effect in patients with sepsis and continuous infusion of piperacillin/tazobactam with regard to the mean SOFA score. Larger studies with strategies to ensure optimization of antimicrobial exposure are needed to definitively answer the question.

Keywords: Sepsis, Therapeutic drug monitoring, $\beta$-Lactams

\section{Introduction}

Sepsis is a life-threatening organ dysfunction caused by a dysregulated host response to infection [1,2]. Emerging evidence suggests that in patients with sepsis, insufficient antibiotic exposure, defined as failure to meet the pharmacokinetic/pharmacodynamic (PK/PD) target to kill or inhibit the growth of a pathogen, is associated with worse clinical outcomes [3-5]. Previous studies demonstrated that up to $50 \%$ of critically ill patients receiving a $\beta$-lactam antibiotic with regimens based on the manufacturers' recommendations fail to reach the target [3, 6]. Reasons for insufficient antibiotic exposure include both altered pharmacokinetics driven by the pathophysiological changes associated with critical illness and/or reduced antibiotic susceptibility of the pathogen [7]. One approach to optimize exposure to $\beta$-lactam antibiotics is administration by prolonged (continuous or extended) infusion [8]. Yet, even with this alternative dosing strategy, not all patients achieve sufficient antibiotic exposure $[9,10]$. An additional intervention to further optimize $\mathrm{PK} / \mathrm{PD}$ target attainment of $\beta$-lactam antibiotics is therapeutic drug monitoring (TDM)-guided therapy [11]. However, although there is a strong precedent for TDM to optimize dosing for antibiotics with a narrow therapeutic index to minimize the likelihood of toxicity (e.g., aminoglycosides, glycopeptides) $[12,13]$ limited data are available regarding the role of TDM of $\beta$-lactam antibiotics. Available studies demonstrated an increased PK/PD target attainment when TDM for piperacillin/tazobactam was performed, however none of the studies reported a difference on clinical outcomes [14-18]. Furthermore, only two of the studies have been randomized controlled trials. The study by De Waele et al. [14] enrolled 28 critically ill patients and the study by Sime et al. [18] enrolled 32 patients with febrile neutropenia and piperacillin/ tazobactam therapy. Both studies reported better target attainment in patients with TDM-guided therapy, however the small sample size precluded to draw significant conclusions on clinical outcomes. Given the abovementioned limitations of previous studies, we aimed to form a more definitive conclusion on whether TDM of piperacillin/tazobactam can improve clinical outcome in patients with sepsis by means of a randomized clinical trial with a large sample size. This trial tested the hypothesis that a TDM-based dose optimization compared to fixed

\section{Take-home message}

In this multicenter, randomized, controlled trial we did not observe a significant benefit for TDM-guided therapy in adult patients with sepsis and continuous infusion of piperacillin/tazobactam with regard to the primary endpoint, namely, the mean total SOFA score. Further studies are necessary to identify patients which might benefit most from a TDM-guided $\beta$-lactam antibiotic therapy and to confirm the observed benefit in 28-day mortality, as well as clinical and microbiological cures, for which the current study was not powered.

dosing would improve clinical outcome in patients with sepsis treated with piperacillin/tazobactam continuous infusion.

\section{Methods \\ Study design and inclusion criteria}

We performed a randomized, multicenter, patient blinded, controlled trial including adult patients with severe sepsis or septic shock in whom therapy with piperacillin/tazobactam was initiated by the treating physician. Patients were eligible for study enrollment if sepsis onset and start of piperacillin/tazobactam therapy occurred no more than $24 \mathrm{~h}$ prior to randomization. Sepsis was defined according to the criteria valid at the time of initiation of the study (Sepsis-2 criteria) [19]. The trial protocol has been published previously [20], and the trial is registered at the German Clinical Trials Register (DRKS00011159; 10th October 2016). Exclusion criteria were contraindications to the study drug, impaired liver function (Child-Pugh C), palliative care only, pregnancy/ lactation, participation in another clinical trial, and first measurement of piperacillin concentration not possible within $24 \mathrm{~h}$ after randomization. Clinical, microbiological, and laboratory data were recorded daily from day before randomization until day 14, at the end of therapy, at discharge from the intensive care unit (ICU) and at day 28. Site investigators used standardized definitions to determine the final clinical and microbiological outcomes [20]. In addition, the Intensive Care Delirium Screening Checklist (ICDSC) was performed regularly [21]. Written informed consent was obtained from each patient or his or her legal representative. Use of antimicrobial combination therapy, termination or (de-) escalation of antimicrobial therapy was allowed at any time and at the discretion of the treating physicians. Antimicrobial 
susceptibility testing was performed by the local microbiology laboratory. The current EUCAST breakpoints for susceptibility were used to classify the susceptibility of isolates. The institutional review board of each study site and Germany's Federal Institute for Drugs and Medical Devices (EudraCT: 2016-000136-17, ref: 4041358) approved the protocol.

\section{Study population, stratification and randomization}

Patients were screened for enrollment at 13 sites in Germany from January 2017 to December 2019 (eTable 1 electronic supplementary material). Patients were randomly assigned (1:1) to the experimental therapy arm (TDM) or to the control group (no-TDM). Randomization was stratified by the participating centers and performed by the investigators using an Internet-based randomization tool [20].

\section{Intervention}

\section{Experimental therapy arm}

After randomization, a continuous infusion of piperacillin/tazobactam, corresponding to a total daily dose of $13.5 \mathrm{~g}$ [9 g in patients with an estimated glomerular filtration rate $(\mathrm{eGFR})<20 \mathrm{~mL} / \mathrm{min}$ ], was started immediately after administration of a loading dose of $4.5 \mathrm{~g}$ piperacillin/tazobactam. Beginning on day 1 after randomization (optional on day 0 , if steady state of piperacillin concentration was already reached), dosing of piperacillin/ tazobactam was guided by daily TDM of piperacillin. The target plasma concentration of free piperacillin was defined as four times (range $\pm 20 \%$ ) the minimal inhibitory concentration (MIC) of the pathogen causative of sepsis. For empiric therapy, the epidemiological cutoff (ECOFF) published by the European Committee on Antimicrobial Susceptibility Testing (EUCAST) of Pseudomonas aeruginosa (16 mg/L) was used (eTable 2a electronic supplementary material). This cutoff value represents a worst-case scenario for empirical dosing. Piperacillin doses not in target were adjusted linearly with taking account for other clinical parameters, e.g., recovering renal function. The maximum duration of the intervention was 10 days.

\section{Control arm}

After randomization continuous infusion of piperacillin/ tazobactam was administered precisely as in the intervention group but without use of TDM. During piperacillin/tazobactam therapy, daily dose adjustments were performed according to current renal function, assessed by eGFR, according to the recommendations of the Summary of Product Characteristics or use of renal replacement therapy (eTable 2b electronic supplementary material).

\section{Sample analysis}

Blood samples for measurement of piperacillin concentration were obtained daily in both study groups. Analysis, reporting of the results and adjustments of doses were performed on the same day only in the TDM group. In the control group, analysis was performed on the same day or later with samples stored at $-80^{\circ} \mathrm{C}$ until analysis. On-site measurements of the total piperacillin concentration were performed in study centers with either high-performance liquid chromatography (HPLC) or liquid chromatography mass spectrometry (LC-MS/ MS) following the guidelines of the Bioanalytical Method Validation of the European Medicines Agency [22]. An external interlaboratory proficiency testing program was performed regularly with participating laboratories.

\section{Outcome measures and adverse events}

The primary endpoint was sepsis-related organ dysfunction measured by the mean daily total sequential organ failure assessment (SOFA) scores over 10 days, discharge from the ICU or death, whichever occurred first. The mean SOFA score was calculated as the mean of all daily SOFA scores for each patient [23]. Secondary outcomes were 28-day all-cause mortality; mean SOFA subscores; duration of ICU and hospital stay; clinical and microbiological treatment response; intervention-free days with a ventilator, vasopressor, dialysis, or antibiotic; $\mathrm{PK} / \mathrm{PD}$ indices; ICDSC, and adverse events.

\section{Sample size calculation}

The study was planned to detect a difference of 1.4 points in the mean SOFA score between the 2 intervention groups with a significance level of 0.05 and a power level of $80 \%$. Data from previous trials showed that such an effect is clinically relevant [24]. Assuming an SD of 3.8 points, 234 patients were required (nQuery Advisor 7.0 Statistical Solutions). Accounting for an expected dropout rate of $15 \%, 276$ patients were planned to be randomized. However, the dropout rate was lower than expected, and study enrollment was stopped after randomization of 254 patients.

\section{Statistical analysis}

A baseline-adjusted linear mixed model was fitted for the primary endpoint, namely, the mean total SOFA score with treatment and renal insufficiency/expected renal replacement as fixed effects and the study center as a random effect. The main effect of the intervention was tested in a confirmatory analysis at the two-sided significance level of $5 \%$ in the intention-to-treat population. Additionally, the same model was applied for the total SOFA score at day 10 (or at earlier discharge from ICU) to explore the sensitivity regarding different 


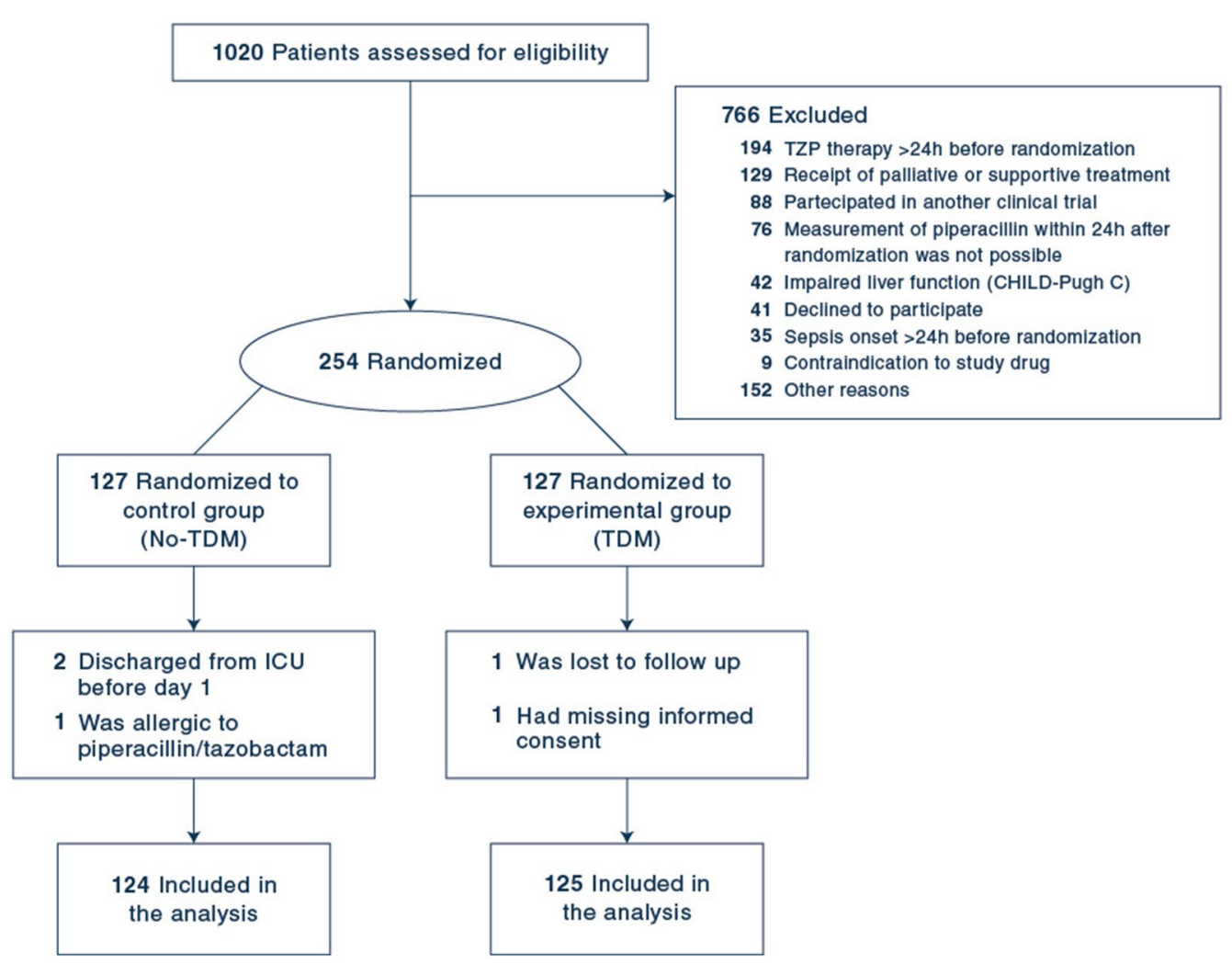

Fig. 1 Flowchart of patient recruitment, inclusion and randomization. TZP = piperacillin/tazobactam; $/ C U=$ intensive care unit

definitions (mean total SOFA score day 1-day 10, total SOFA score at day 10) of the endpoint. 28 days all-cause mortality were compared by means of Fisher's exact test (two sided), and relative risk with 95\% confidence interval as well as group-wise absolute and relative frequencies are provided. Furthermore, Kaplan-Meier curves are provided for this endpoint and the log rank test was performed to compare the survival curves. SOFA subscores, length of stay (ICU, hospital), intervention-free days, total daily dose of piperacillin/tazobactam, duration of therapy with piperacillin/tazobactam, piperacillin concentrations up to day 10 , overall duration of antibiotic therapy were compared by independent samples $t$ test in case of normally distributed data or the Mann-Whitney $U$ test otherwise, and the mean \pm SD (standard deviation) or median and interquartile range are reported for both groups. $Q-Q$ plots were used to check if the data are normally distributed. Generalized linear mixed models were fitted for neurological outcome per ICDSC, clinical and microbiological cure and attainment of target concentration (fixed effects: treatment and visit including their interaction; random effect: site) and odds ratios with $95 \%$ confidence intervals are reported. To analyze the relationship of piperacillin exposure and 28-day mortality a binary logistic regression model was fitted for mortality with piperacillin concentration and treatment as independent variables, adjusted for baseline SOFA score. Odds ratios with $95 \%$ confidence intervals are reported for different levels of piperacillin concentration. Analyses were performed using SAS 9.4 (SAS Institute, Cary NC).

\section{Results}

A total of 1020 patients were screened during the trial period. Of those, $254(24.9 \%)$ patients were randomized (Fig. 1). In the TDM group, one patient was excluded due to a missing informed consent form, and one patient was lost to follow-up. In the control group, two patients were discharged from the ICU before day 1 and were therefore excluded from the analysis, and piperacillin/tazobactam was stopped after its first application in one patient due to a possible allergic reaction. After these exclusions, 249 patients, including 124 patients in the control group and 125 patients in the TDM group, were included in the final analysis.

\section{Demographic and clinical characteristics of the patients}

Mean age of patients was $66.3 \pm 13.7$ years and 30.9\% of patients were female. Overall, $74.3 \%$ of patients were diagnosed with septic shock (Sepsis-2 definition). When applying ex post the currently valid Sepsis-3 definition 
[2], 52.2\% of patients fulfilled the criteria for the diagnosis of septic shock and the remaining $47.8 \%$ the definition for sepsis. Overall, the treatment groups were well balanced with respect to baseline characteristics (Table 1). Concerning antimicrobial combination therapy there was also no difference between study groups. On day one, $38.5 \%$ of patients in the TDM group and $42.9 \%$ of patients without TDM received an additional antibiotic agent. The most common combination partner in both groups was a fluoroquinolone (28.1\% of patients), followed by vancomycin (14.4\%), macrolides (13.6\%) and linezolid (10.8\%). In 166 of 253 study participants (65.6\%), a causative pathogen was identified. The most common pathogen was Escherichia coli $[n=86$ (17.0\%), followed by Klebsiella spp. $(n=59(11.7 \%)]$ and methicillin-susceptible Staphylococcus aureus $[n=34$ (6.7\%)] (eTable 3 electronic supplementary material). The piperacillin/tazobactam MIC of the five most commonly detected Gram-negative pathogens, which represent 78\% (228/290) of all detected Gram-negative bacteria and $45 \%$ (228/505) of all detected pathogens, was $\leq 4 \mathrm{mg} / \mathrm{L}$ in $79.8 \%(182 / 228)$ of isolates. Only $6.1 \%(14 / 228)$ of isolates had a MIC of $8-16 \mathrm{mg} / \mathrm{L}$ and $14.0 \%(32 / 228)$ of isolates were resistant to piperacillin/tazobactam (MIC > $16 \mathrm{mg} / \mathrm{L}$ ) (eTable 4 electronic supplementary material). Median time to availability of MIC values in patients with TDM-guided therapy was 2 days (2-3).

\section{Primary and secondary outcomes}

The mean SOFA score was 7.9 points [95\% confidence interval (CI), 7.1-8.7] in the TDM group and 8.2 points (95\% CI 7.5-9) in the control group, with no significant difference $(0.3$ points, $95 \% \mathrm{CI}-0.4$ to $1, p=0.39$ ) (efigure 1 electronic supplementary material). Likewise, there was no difference in delta SOFA score, either measured as difference between score at day 10 (or discharge from ICU) minus score at baseline, nor if in patients deceased within 10 days after randomization the last SOFA score was set to the maximum value of 24 points to account for a survivorship bias (Table 2). All-cause mortality at 28 days was $21.6 \%$ in the TDM group and $25.8 \%$ in patients in the control group (RR 0.8, 95\% CI 0.5-1.3, $p=0.44$ ). KaplanMeier survival curves showed no significant differences between both treatment groups (log rank test $p=0.49$ ) (Fig. 2). In addition, we observed no significant difference for any other secondary outcome, albeit patients with TDM showed a higher rate of clinical (OR 1.9; 95\% CI 0.5-6.2, $p=0.30$ ) and microbiological cure (OR 2.4; 95\% CI 0.7-7.4, $p=0.12$ ) during therapy (Table 2). Mean treatment duration with piperacillin/tazobactam (TDM $4.8 \pm 3$ days vs. no-TDM $4.8 \pm 2.8$ days, $p=0.81)$ and overall duration of antibiotic therapy
(TDM $6.8 \pm 10$ days vs. no-TDM $6.6 \pm 8.7$ days, $p=0.7$ ) were similar between both treatment groups. In addition, there was no difference of the mean total daily dose of piperacillin/tazobactam administered (TDM $10.3 \mathrm{~g} \pm 5.6 \mathrm{vs}$. no-TDM $9.8 \mathrm{~g} \pm 2.5, p=0.12$ ) (eFigure 2 electronic supplementary material). Finally, no difference was observed in the neurological outcome per Intensive Care Delirium Screening Checklist (ICDSC) (OR 1.3; 95\% CI 0.4-4.8, $p=0.68$ ).

\section{Study intervention}

In the TDM group, 579 piperacillin concentration measurements were required in total. Of those, $88.1 \%$ $(n=510)$ were performed on the same day, $10.0 \%$ $(n=58)$ were performed at a later date and $1.9 \%$ $(n=11)$ have never been performed. In the control group, 600 measurements were required in total, of those $2 \%(n=12)$ have never been performed. Overall, in $53.9 \%(312 / 579)$ of days with piperacillin/tazobactam therapy in patients with TDM-guided therapy a dose adjustment was performed, in $51.2 \%$ an increase and in $48.8 \%$ a decrease in dose was required (eTables 5 and 6 electronic supplementary material). In contrast, in patients without TDM, a dose adjustment was performed on only $5.5 \%(33 / 600)$ of days with piperacillin therapy. No significant difference was observed in the median piperacillin concentrations up to day 10 [TDM $74.9 \mathrm{mg} / \mathrm{L}$ (60.6-91) vs. no-TDM $79 \mathrm{mg} / \mathrm{L}$ (43.8-143.6), $p=0.38$ )] between the study groups (eFigure 3 electronic supplementary material).

In both study groups the most common target for piperacillin concentration was $80 \mathrm{mg} / \mathrm{L}$ in $73.7 \%$ (869/1179) of days with piperacillin/tazobactam therapy, followed by a target of $20 \mathrm{mg} / \mathrm{L}$ for $22.2 \%$ (262/1179) of days and $40 \mathrm{mg} / \mathrm{L}$ for $4.1 \%$ (48/1179) of days. Attainment of target concentration on individual days was more often achieved in the TDM group $(37.3 \%$ vs. $14.6 \%$, OR 4.5, CI 95\%, 2.9-6.9, $p<0.001$ ) (Fig. 3, eTable 7 electronic supplementary material). Consequently, the risk of piperacillin concentrations below (OR 0.6, CI 95\%, 0.3$1.2, p<0.17$ ) or above (OR 0.5, CI 95\%, 0.2-0.9, $p<0.02$ ) the target range was lower in patients with TDM-guided therapy.

\section{8-day mortality based on piperacillin concentration and clearance}

To investigate a probable relationship between piperacillin exposure in the early phase of sepsis and outcome, we analyzed the piperacillin concentration on day 1 after randomization and corresponding 28-day mortality. After adjustments were made for disease severity according to the baseline SOFA score, the 28-day mortality rate of patients with a piperacillin concentration $>96 \mathrm{mg} / \mathrm{L}$ 
Table 1 Demographics and baseline characteristics

\begin{tabular}{|c|c|c|}
\hline Characteristic & TDM $(n=125)$ & No-TDM $(n=124)$ \\
\hline Age, mean (SD), years & $67.2(13.9)$ & $65.3(13.5)$ \\
\hline Male sex, no. (\%) & $80(63.5)$ & $92(72.4)$ \\
\hline Body mass index, mean (SD) ${ }^{a}$ & $28.3(7.9)$ & $27.4(7.4)$ \\
\hline APACHE II score, mean (SD) ${ }^{b}$ & $23.2(6.7)$ & $22.4(5.7)$ \\
\hline SOFA score, mean $(\mathrm{SD})^{\mathrm{C}}$ & $12.1(2.8)$ & $12.2(2.6)$ \\
\hline SAPS II score, mean (SD) ${ }^{b}$ & $44.6(12.4)$ & $43.9(12.2)$ \\
\hline Charlson comorbidity index score, median (IQR) & $2(1-3)$ & $2(1-3)$ \\
\hline Septic shock, no. (\%) & $96(76.2)$ & $92(72.4)$ \\
\hline Required mechanical ventilation, no. (\%) & $100(79.3)$ & $92(72.4)$ \\
\hline \multicolumn{3}{|l|}{ Laboratory values, median (IQR) } \\
\hline White blood cell count, cells/ $\mu \mathrm{L}$ & $17.0(11.7-22.2)$ & $13.6(10-23.5)$ \\
\hline Plasma procalcitonin, ng/mL & $4.3(0.9-13.4)$ & $4.2(1.0-14.5)$ \\
\hline Plasma lactate, mg/dL & $2.2(1.5-3.5)$ & $2.2(1.4-3.6)$ \\
\hline Plasma creatinine, $\mathrm{mg} / \mathrm{dL}$ & $1.3(0.84-2)$ & $1.4(0.9-2.3)$ \\
\hline Creatinine clearance, $\mathrm{mL} / \mathrm{min}$ & $55.6(34.5-90.3)$ & $53(32.7-95)$ \\
\hline Plasma albumin, g/dL & $2.5(2.2-2.9)$ & $2.4(2-3)$ \\
\hline \multicolumn{3}{|l|}{ Source of infection, no. (\%) } \\
\hline Pneumonia & $74(62.7)$ & $81(65.8)$ \\
\hline Intra-abdominal infection & $25(21.2)$ & $24(19.5)$ \\
\hline Urinary tract & $15(12.7)$ & $17(13.8)$ \\
\hline Bone or soft tissue & $11(9.3)$ & $15(6.2)$ \\
\hline Surgical site infection & $5(4.2)$ & $4(3.3)$ \\
\hline Other & $20(16.9)$ & $17(13.8)$ \\
\hline Unknown & $8(6.3)$ & $4(3.1)$ \\
\hline \multicolumn{3}{|l|}{ Acquisition, no. (\%) } \\
\hline Health care-associated & $71(56.3)$ & $72(56.7)$ \\
\hline Community-associated & $55(43.7)$ & $55(43.3)$ \\
\hline Time between onset of sepsis and randomization, mean (SD), $\mathrm{h}$ & $15.0 \pm 6.4$ & $15.1 \pm 6.9$ \\
\hline \multicolumn{3}{|c|}{$\begin{array}{l}\text { SI conversion factors: To convert plasma albumin from } \mathrm{g} / \mathrm{dL} \text { to } \mathrm{g} / \mathrm{L} \text {, multiply by } 1.0 ; \text { plasma procalcitonin from } \mathrm{ng} / \mathrm{mL} \text { to } \mu \mathrm{g} / \mathrm{L} \text {, } \mathrm{multiply} \text { by } 1.0 ; \text { plasma creatinine from } \\
\mathrm{mg} / \mathrm{dL} \text { to } \mu \mathrm{mol} / \mathrm{L} \text {, multiply by } 88.4 \text {; creatinine clearance from } \mathrm{mL} / \mathrm{min} \text { to } \mathrm{mL} / \mathrm{s} \text {, multiply by } 0.0167 \text {; plasma lactate from } \mathrm{mg} / \mathrm{dL} \text { to } \mathrm{mmol} / \mathrm{L}, \mathrm{multiply} \mathrm{by} 0.111\end{array}$} \\
\hline \multicolumn{3}{|l|}{ APACHE Acute Physiology and Chronic Health Evaluation, IQR interquartile } \\
\hline \multicolumn{3}{|l|}{ a Calculated as weight in kilograms divided by height in meters squared } \\
\hline \multicolumn{3}{|l|}{ b Missing subscores were counted as 0} \\
\hline \multicolumn{3}{|c|}{ c The scale score ranges from 0 to 24 , with higher scores indicating a greater severity of organ failure } \\
\hline d Multiple responses per patient possible & & \\
\hline
\end{tabular}

(35/104) was significantly higher than that of patients with a concentration of $32-64 \mathrm{mg} / \mathrm{L}(4 / 48)(33.7 \%$ vs. $8.3 \%$, OR $4.21,95 \%$ CI $1.4-12.5, p=0.01)$ or $64-96 \mathrm{mg} / \mathrm{L}$ (12/61) (33.7\% vs. $19.7 \%$, OR 2.5, 95\% CI 1.1-5.8, $p=0.03)$ The piperacillin clearance rate was significantly different among these groups, with the lowest clearance rate observed for patients with a piperacillin concentration $>96 \mathrm{mg} / \mathrm{L}(2.2 \mathrm{~mL} / \mathrm{min})$ and the highest clearance rate observed for patients with a concentration $<16 \mathrm{mg} / \mathrm{L}$ (29.3 $\mathrm{mL} / \mathrm{min}$ ) (eFigure 4 electronic supplementary material). When adjusting for APACHE II score, patients achieving the individual piperacillin target within the first two days showed a better resolution of organ dysfunction compared to patients who did not achieve target attainment (difference in mean total SOFA score of day 1 and day 2 : $-1.2,95 \% \mathrm{CI}-2.2$ to $-0.1, p=0.02$ ).

\section{Adverse events}

Twenty-five adverse events occurred in 20 patients (15.7\%) in the TDM group, and 33 adverse events occurred in 27 patients $(21.3 \%)$ in the control group $(p=0.24)$. The most often reported adverse event was hypernatremia, with 12 reported episodes in the TDM group and 13 episodes in the control group, followed by gastrointestinal symptoms, with 6 events reported in the control group and 3 events reported in the TDM group. 
Table 2 Study outcomes

\begin{tabular}{|c|c|c|c|}
\hline Outcome & $\mathrm{TDM}(n=125)$ & No-TDM $(n=124)$ & $p$ value $^{a}$ \\
\hline SOFA score, mean (95\% Cl) & $7.9(7.1-8.7)$ & $8.2(7.5-9)$ & 0.39 \\
\hline 28-Day mortality, no. (\%) & $27(21.6)$ & $32(25.8)$ & 0.44 \\
\hline $\begin{array}{l}\triangle S O F A \text {, mean score day } 1-10 \text { (or } 24 \text { points if death within } 10 \text { days) minus } \\
\text { score at baseline }\end{array}$ & $2.1(-0.2-4.3)$ & $2.6(0.3-4.9)$ & 0.59 \\
\hline $\begin{array}{l}\triangle S O F A \text {, score at day } 10 \text { (or } 24 \text { points if death within } 10 \text { days) minus score } \\
\text { at baseline }\end{array}$ & $1.6(-1-4.2)$ & $2.9(0.2-5.6)$ & 0.26 \\
\hline \multicolumn{4}{|l|}{ SOFA subscore, median (IQR) } \\
\hline Cardiovascular & $2(1-3)$ & $2(1.2-3.2)$ & 0.81 \\
\hline Respiratory & $2.5(2-3)$ & $2.5(2-2.9)$ & 0.45 \\
\hline Coagulation & $0.1(0-1)$ & $0(0-0.8)$ & 0.54 \\
\hline Renal & $0.5(0-1.5)$ & $0.8(80-2)$ & 0.4 \\
\hline Hepatic & $3.2(2.6-4)$ & $3.3(2.8-4)$ & 0.68 \\
\hline Central nervous system & $0.1(0-1.2)$ & $0.3(0-1.3)$ & 0.31 \\
\hline \multicolumn{4}{|l|}{ Length of stay (days), median (IQR) } \\
\hline $\ln I C U$ & $9(4-15)$ & $11(7-17$ & 0.24 \\
\hline In hospital & $24(15-28)$ & $25(15-28)$ & 0.52 \\
\hline \multicolumn{4}{|l|}{ Intervention-free days, median (IQR) } \\
\hline Ventilator $^{b}$ & $20(5-27)$ & $18.5(1-25)$ & 0.06 \\
\hline Renal replacement therapy ${ }^{b}$ & $28(21-28)$ & $28(10-28)$ & 0.33 \\
\hline Antibiotic ${ }^{c}$ & $8(6-12)$ & $8(5-11)$ & 0.19 \\
\hline Vasopressor ${ }^{c}$ & $11(2-13)$ & $9(2-12)$ & 0.14 \\
\hline Clinical cure, EOT ${ }^{d}$ & $21 / 59(35.6)$ & $12 / 69(17.4)$ & \\
\hline Microbiological cure, EOT ${ }^{e}$ & $27 / 48(56.3)$ & $23 / 50(46)$ & \\
\hline Total daily dose (grams) of piperacillin/tazobactam, mean (SD) & $10.3 \pm 5.6$ & $9.8 \pm 2.5$ & 0.12 \\
\hline \multicolumn{4}{|c|}{ a Calculated using the independent samples $t$ test or Mann-Whitney test for continuous outcomes or Fisher's exact test for categorical outcomes } \\
\hline \multicolumn{4}{|l|}{ b Until ICU discharge or day 28 (maximum) } \\
\hline \multicolumn{4}{|l|}{ c Until ICU discharge or day 14 (maximum) } \\
\hline \multicolumn{4}{|c|}{${ }^{d}$ EOT: end of therapy, no. of patients/total no. evaluable patients (\%), No clinical cure was defined as "improvement or treatment failure" } \\
\hline \multicolumn{4}{|c|}{$\begin{array}{l}\text { eOT: end of therapy, no. of patients/total no. evaluable patients (\%), Microbiological cure was defined as either "documented or suspected microbiological } \\
\text { eradication" }\end{array}$} \\
\hline
\end{tabular}

Overall, 7 serious adverse events occurred in the TDM group, three of which were fatal. In the control group, 8 serious adverse events occurred, with 3 fatal outcomes. None of the serious adverse events were judged to be related to the study drug (eTable 8 electronic supplementary material).

\section{Discussion}

In this clinical trial with 249 patients with sepsis, we observed no significant beneficial effect of TDMguided piperacillin/tazobactam therapy with regard to the 10-day mean total SOFA score compared to fixed dosing. However, in patients who underwent TDMguided therapy we observed a $4.2 \%$ lower 28 -day mortality and a higher rate of microbiological and clinical cure during therapy, but these differences were not statistically significant. Similarly, attainment of the target piperacillin concentration was significantly more often achieved in patients with TDM-guided therapy, and the risk of underdosing was higher in patients with fixed dosing. Furthermore, we confirmed the previously described high variability of piperacillin concentrations in critically ill patients with sepsis [3]. Considering all days with piperacillin/tazobactam therapy, a dose adjustment was required in approximately half of the days to reach the target concentration in patients with TDM-guided therapy. Nevertheless, attainment of the target concentration on individual days was achieved in one-third of patients with TDM only. This reflects the fact that critically ill patients with sepsis have highly dynamic pharmacokinetics, and therefore, it is difficult to hit the exact target range, even with daily TDM. In a study from De Wale et al. [14], only just over half of the patients achieved target attainment despite daily TDM. However, in contrast to our study, with a lower and upper threshold defining the piperacillin target concentration, De Wale et al. defined target attainment 


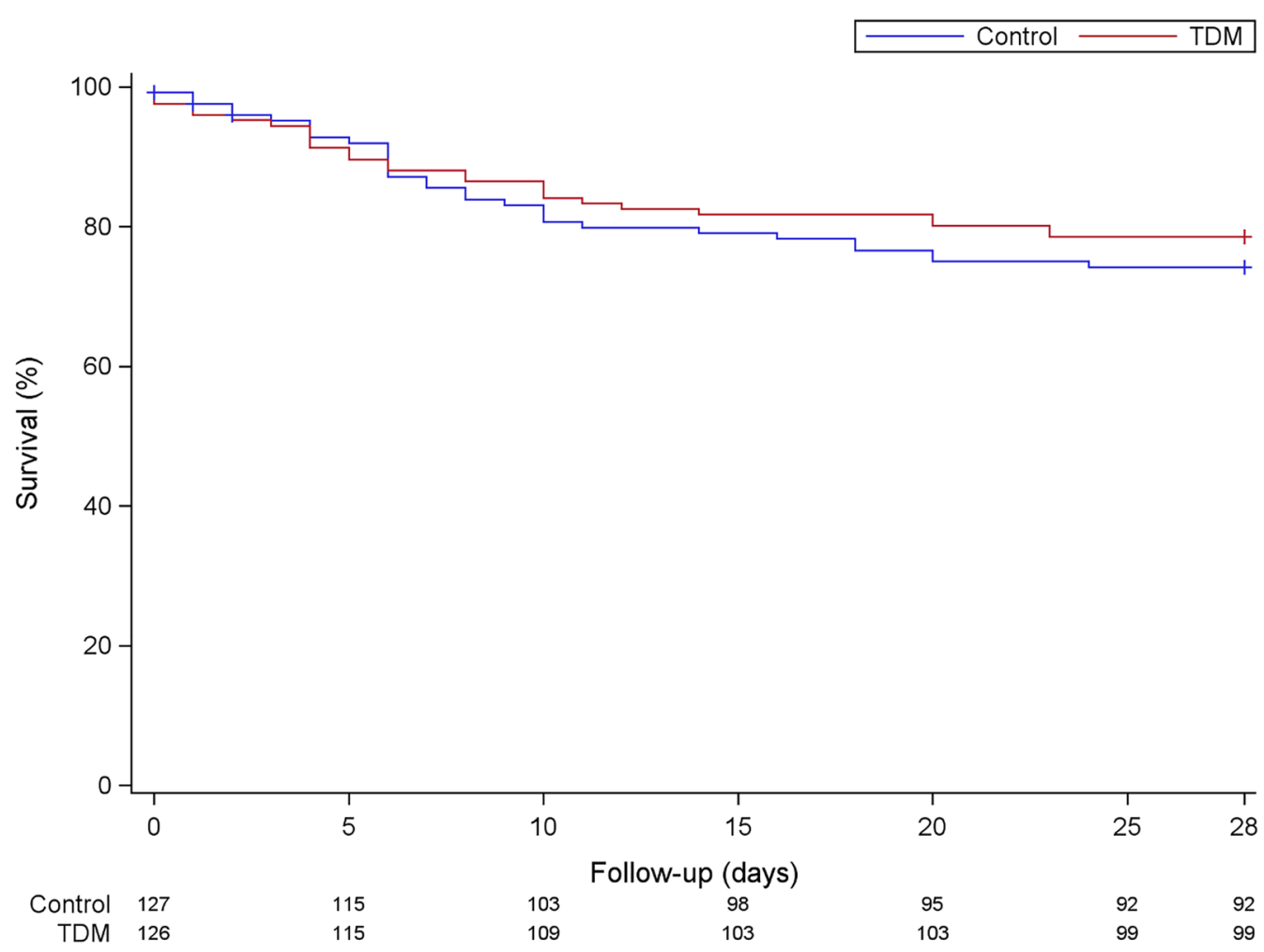

Fig. 2 Kaplan-Meier analysis. Overall survival rates at day 28 among patients with piperacillin TDM-guided therapy (TDM) and patients in the control group (control). Number of patients at risk for each group included in the analysis along the $x$-axis scale

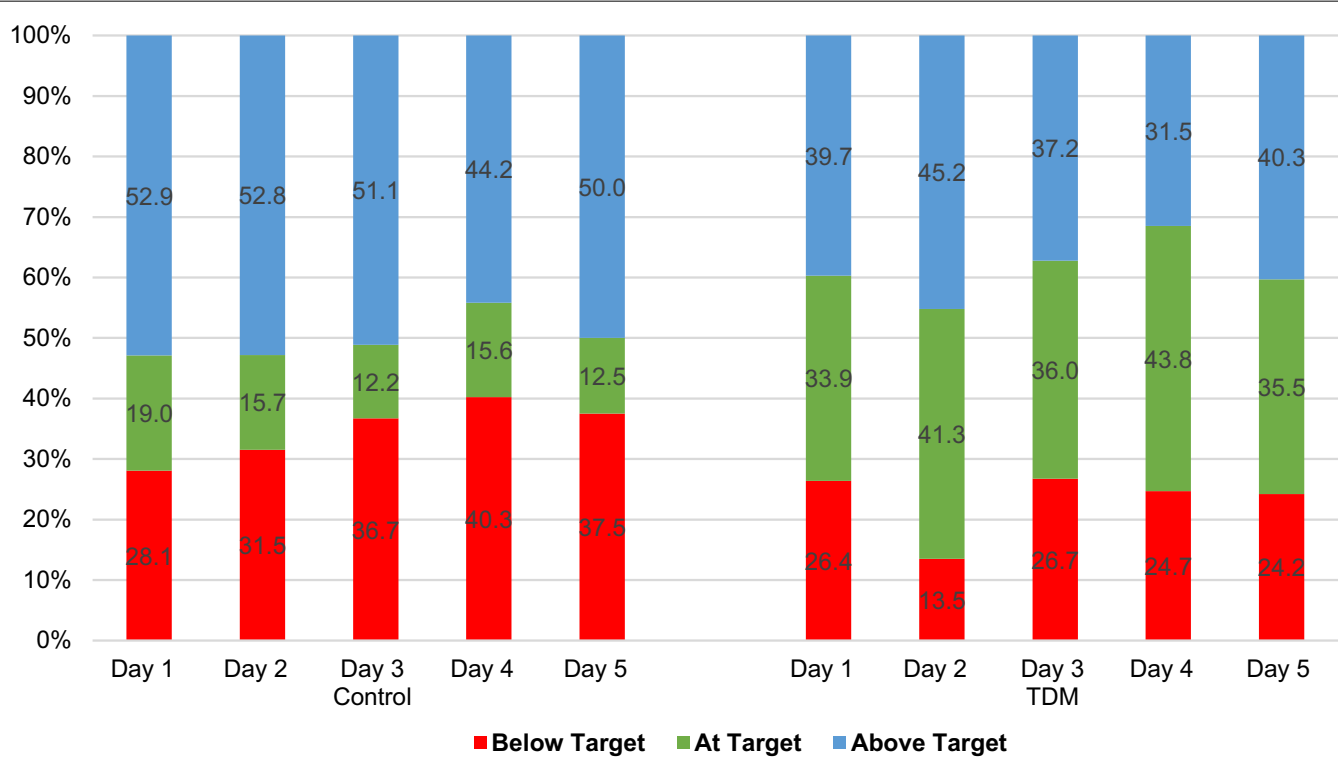

Fig. 3 Attainment of piperacillin target concentration in percent up to day 5 after randomization in patients with piperacillin TDM-guided therapy (TDM, right columns) and patients in the control group (control, left column). Within range (green), above range (blue) or below range (red) per the target range on a given day 
as a concentration $>4$ times the MIC, irrespective of the concentration.

It is unclear to what extent the overall results in our study were influenced by the low rate of target attainment. For example, in nearly one-third of patients, the commonly suggested target concentration in empiric situations, namely, four times the ECOFF of $P$. aeruginosa, was not reached on the first day after randomization. However, this was not associated with a worse clinical outcome. This finding might reflect the fact that the majority of bacteria identified within the study displayed a low MIC within the susceptible range; therefore, supposedly insufficient piperacillin concentrations, designated as "underdosed", were still adequate for successful antimicrobial therapy.

In contrast, patients with a piperacillin concentration above the maximum target had the highest 28-day mortality in our study. The same was observed in a retrospective study by Richter et al. [16] that included 484 patients with sepsis who received TDM-guided continuous infusion of piperacillin/tazobactam. In this study, patients with a piperacillin concentration of $\geq 64 \mathrm{mg} / \mathrm{L}$ within the first $24 \mathrm{~h}$ exhibited a higher mortality than patients with a concentration of $33-64 \mathrm{mg} / \mathrm{L}(29.4 \%$ vs. $13.9 \%, p<0.005)$. This increase in mortality observed among patients with higher piperacillin concentrations is most likely a consequence of pronounced sepsis-associated organ dysfunction-most importantly, loss of renal function-which leads to a decreased piperacillin clearance and hence piperacillin accumulation, as shown in our study.

Interestingly, when adjusting for APACHE II score, patients achieving the individual piperacillin target concentration within the first two days showed a better resolution of organ dysfunction compared to patients who did not achieve target attainment. Above all, an independent increase in mortality or otherwise associated toxicity of excessive piperacillin exposure cannot be excluded.

\section{Limitations}

This study has several limitations. First, no dosage algorithm or dosing software was used to support consistent dosing adjustments in patients with TDM-guided therapy. With such tools, there might have been further optimization for target attainment. Second, the caregivers were not blinded to the study groups, which in turn might have influenced the treatment of patients in the control group, e.g., escalation of antibiotic therapy more often. However, we did not observe a significant difference in the duration of therapy with piperacillin/ tazobactam in either study group; therefore, this might constitute a rather small bias. Third, as the methodology can vary according to the laboratory, as well as because of biological variability, it is important to acknowledge the limitations of MIC values, which can range between 1 and 2 dilutions [25]. Fourth, only serum piperacillin concentrations were obtained, and these concentrations do not always reflect concentrations at the site of infection [26]. Furthermore, the study was performed in a single country with a low prevalence of resistant gram-negative bacteria, which limits the generalizability of our results. Finally, antibiotic combination therapy was allowed at any time, which might have biased the study results. However, excluding patients with concomitant fluoroquinolone therapy did not show any difference in outcome between patients with TDM and fixed doses (data not shown).

\section{Conclusions}

In conclusion, we did not observe a significant benefit for TDM-guided therapy in patients with sepsis and continuous infusion of piperacillin/tazobactam with regard to the primary endpoint, namely, the mean total SOFA score. Studies with a higher number of patients are necessary to confirm the observed benefit in 28-day mortality, as well as clinical and microbiological cures, for which the current study was not powered. In addition, further studies are necessary to identify patients who might benefit most from TDM-guided therapy with $\beta$-lactam antibiotics, such as patients with an augmented renal clearance or patients with bacterial infections that demonstrate higher MICs.

\section{Data sharing}

With publication, deidentified, individual participant data that underlie this article, along with a data dictionary describing variables in the dataset, will be made available to researchers whose proposed purpose of use is approved by the Trial Management Team. To request the dataset, please address directly to the corresponding author to obtain a data access form. All requests will be evaluated by the Trial Management Team. For accepted requests, data will be shared after signing a data transfer agreement with the study sponsor. Related documents, such as the study protocol, statistical analysis plan, and informed consent form, will be made available (with publication) on request to the corresponding author and open access.

\section{Supplementary Information \\ The online version contains supplementary material available at https://doi. org/10.1007/s00134-021-06609-6.}

\section{Author details}

${ }^{1}$ Institute for Infectious Diseases and Infection Control, Jena University Hospital-Friedrich Schiller University Jena, Am Klinikum 1, 07747 Jena, Germany. 
${ }^{2}$ Center for Sepsis Control and Care (CSCC), Jena University Hospital-Friedrich Schiller University Jena, Jena, Germany. ${ }^{3}$ Department of Infectious Diseases, Evangelisches Klinikum Bethel, Bielefeld, Germany. ${ }^{4}$ Department of Anesthesiology and Intensive Care Medicine, University Hospital Essen, University Duisburg-Essen, Essen, Germany. ${ }^{5}$ Department of Anesthesiology, Heidelberg University Hospital, Heidelberg, Germany. ${ }^{6}$ Department of Anaesthesiology and Critical Care Medicine, University Hospital of UIm, UIm, Germany. ${ }^{7}$ Department of Anaesthesiology and Intensive Care Medicine, General Hospital of Heidenheim, Heidenheim, Germany. ${ }^{8}$ Department of Anaesthesiology and Intensive Care Medicine, Faculty of Medicine, University of Cologne, Faculty of Medicine and University Hospital of Cologne, Cologne, Germany. ${ }^{9}$ Department of Anesthesiology and Intensive Care Medicine, Kliniken der Stadt Köln gGmbH, University of Witten/Herdecke, Cologne, Germany.

${ }^{10}$ Department of Anaesthesiology and Intensive Care Medicine, Kliniken Maria Hilf, Mönchengladbach, Germany. ${ }^{11}$ Department of Acute and Emergency Care, St-Antonius-Hospital gGmbH, Eschweiler, Germany. ${ }^{12}$ Department of Intensive Care Medicine, University Medical Centre Hamburg-Eppendorf, Hamburg, Germany. ${ }^{13}$ Medizinische Klinik Und Poliklinik I, Internistische Notfall- und Intensivmedizin, Universitätsklinikum Würzburg, Würzburg, Germany. ${ }^{14}$ Department of Pharmacy, General Hospital of Heidenheim, Heidenheim, Germany. ${ }^{15}$ Centre of Pharmacology, Department of Therapeutic Drug Monitoring, University Hospital of Cologne, Cologne, Germany. ${ }^{16}$ Department of Anesthesiology and Intensive Care Therapy, Jena University HospitalFriedrich Schiller University Jena, Jena, Germany. ${ }^{17}$ Department of Clinical Chemistry and Laboratory Medicine, Jena University Hospital-Friedrich Schiller University Jena, Jena, Germany. ${ }^{18}$ Institute of Medical Statistics, Computer Sciences and Data Sciences, Jena University Hospital-Friedrich Schiller University Jena, Jena, Germany. ${ }^{19}$ University of Queensland Centre for Clinical Research, and School of Pharmacy, University of Queensland, Brisbane, Australia.

${ }^{20}$ Department of Intensive Care Medicine and Pharmacy Department, Royal Brisbane and Women's Hospital, Brisbane, Australia. ${ }^{21}$ Division of Anaesthesiology Critical Care Emergency and Pain Medicine, Nîmes University Hospital, University of Montpellier, Nîmes, France.

\section{Acknowledgements}

The study was funded by the Federal Ministry of Education and Research (BMBF), 01EO1502. In addition, we thank all participants who consented to enrol in the trial, as well as all study and site staff whose indispensable assistance made the conduct of the trial possible.

TARGET Trial Investigators: Anke Braune, Karsten Schmidt, MD, Johann Motsch, MD, Nadine Pinder, Daniel Richter, MD, Peter Schlattmann, MD, Andreas Ameln-Mayerhofer von, Markus Schappacher, MD, Thomas Fuchs, MD, Anka Röhr, Max Kurlbaum, Oliver Schreiner; MD, Lars Hüter, MD, Matthias Gründling, MD, Stefan Angermair, MD, Maria Deja, MD, Frank Bloos, MD, Sandra Fiedler, Hicham Chkirni.

\section{Author contributions}

$S H, A B, M W P, O F, M K, S N, J A R$ and $T L$ were involved in the design of the trial. $S H, F B, T B, H B, A B, T A, A H, M W, G M, S K, A N, D J, D M, D W$ and $M B$ included participants in the trial. $\mathrm{MK}, \mathrm{SN}, \mathrm{OF}, \mathrm{CM}$ and $\mathrm{CK}$ were responsible for the pharmacological analyses. TL and SH were involved in the statistical analyses. SH wrote the original draft of the manuscript. All authors contributed to refinement of and approved this manuscript. All authors had full access to all the data in the study and had final responsibility for the decision to submit for publication.

\section{Funding}

Open Access funding enabled and organized by Projekt DEAL. The study was funded by the Federal Ministry of Education and Research (BMBF), 01 EO1502. The funder had no role in the design; in the collection, analysis, and interpretation of data; in the writing of the report; and in the decision to submit the paper for publication.

\section{Declarations}

\section{Conflicts of interest}

$\mathrm{SH}$ report grants from the Federal Ministry of Education and Research (BMBF), lecture fees from Pfizer, MSD, InfectoPharm, Advanz and Philips, support for attending meetings from Pfizer and Advanz. AB reports lecture fees from MSD Sharp \& Dohme GmbH, Pfizer Pharma GmbH, Fresenius Medical Care (FMC), Labor Volkmann (Karlsruhe). TB reports lecture fees from CSL Behring $\mathrm{GmbH}$, Schöchl medical education GmbH, Biotest AG, Baxter Deutschland
GmbH, Boehringer Ingelheim Pharma GmbH, Astellas Pharma GmbH, B. Braun Melsungen AG, MSD Sharp \& Dohme GmbH, support for participation in a Data Safety and Monitoring Board for Baxter Deutschland GmbH and and research funding from Deutsche Forschungsgemeinschaft (DFG), Dietmar Hopp Stiftung, Stiftung Universitätsmedizin Essen. Heidelberger Stiftung Chirurgie, Innovationsfonds des Gemeinsamen Bundesausschusses (G-BA). SN reports grants from the Center for Sepsis Control and Care (CSCC) by the Ministry of Education and Research (BMBF), Grant-no. 01EO1502. SK reports grants from Ambu, Daiichi Sankyo, ETView Ltd, Fisher \& Paykel, Pfizer and Xenios, consulting fees from Bayer, Fresenius, Gilead, MSD and Pfizer, lecture fees from Astra, C.R.Bard, Baxter, Biotest, Cytosorbents, Daiichi Sankyo, Fresenius, Gilead, Mitsubishi Tanabe Pharma, MSD, Pfizer, Philips and Zoll. MW reports lecture fees from MSD, Gilead, Shionogi, Pfizer, support for participation in a Data Safety and Monitoring Board for MSD, Gilead, Shionogi, Eumedica, Biotest. All other authors declare no competing interests.

\section{Ethics approval}

The trial was approved by the relevant ethics committees in all study sites.

\section{Consent to participate}

Written informed consent was obtained from all patients or from their surrogates.

\section{Consent for publication}

All authors approved the manuscript for submission.

\section{Open Access}

This article is licensed under a Creative Commons Attribution-NonCommercial 4.0 International License, which permits any non-commercial use, sharing, adaptation, distribution and reproduction in any medium or format, as long as you give appropriate credit to the original author(s) and the source, provide a link to the Creative Commons licence, and indicate if changes were made. The images or other third party material in this article are included in the article's Creative Commons licence, unless indicated otherwise in a credit line to the material. If material is not included in the article's Creative Commons licence and your intended use is not permitted by statutory regulation or exceeds the permitted use, you will need to obtain permission directly from the copyright holder. To view a copy of this licence, visit http://creativecommons.org/licen ses/by-nc/4.0/.

\section{Publisher's Note}

Springer Nature remains neutral with regard to jurisdictional claims in published maps and institutional affiliations.

\section{Received: 12 October 2021 Accepted: 21 December 2021} Published online: 1 February 2022

\section{References}

1. Evans L, Rhodes A, Alhazzani W et al (2021) Surviving sepsis campaign: international guidelines for management of sepsis and septic shock 2021. Intensive Care Med. https://doi.org/10.1007/s00134-021-06506-y

2. Singer M, Deutschman CS, Seymour CW et al (2016) The third international consensus definitions for sepsis and septic shock (Sepsis-3). JAMA 315:801-810. https://doi.org/10.1001/jama.2016.0287

3. Roberts JA, Paul SK, Akova M et al (2014) DALI: defining antibiotic levels in intensive care unit patients: are current-lactam antibiotic doses sufficient for critically ill patients? Clin Infect Dis 58:1072-1083. https://doi. org/10.1093/cid/ciu027

4. Lodise TP, Lomaestro B, Drusano GL (2007) Piperacillin-tazobactam for Pseudomonas aeruginosa infection: clinical implications of an extendedinfusion dosing strategy. Clin Infect Dis 44:357-363. https://doi.org/10. 1086/510590

5. Tannous E, Lipman S, Tonna A et al (2020) Time above the MIC of piperacillin-tazobactam as a predictor of outcome in Pseudomonas aeruginosa bacteraemia. Antimicrob Agents Chemother. https://doi.org/10.1128/aac. 02571-19 
6. Taccone FS, Laterre P-F, Dugernier T et al (2010) Insufficient $\beta$-lactam concentrations in the early phase of severe sepsis and septic shock. Crit Care 14:R126. https://doi.org/10.1186/cc9091

7. Huttner A, Harbarth S, Hope WW et al (2015) Therapeutic drug monitoring of the $\beta$-lactam antibiotics: what is the evidence and which patients should we be using it for?: Figure 1. J Antimicrob Chemother. https://doi. org/10.1093/jac/dkv201

8. Roberts JA, Abdul-Aziz M-H, Davis JS et al (2016) Continuous versus intermittent $\beta$-lactam infusion in severe sepsis. A meta-analysis of individual patient data from randomized trials. Am J Respir Crit Care Med 194:681-691. https://doi.org/10.1164/rccm.201601-0024OC

9. Carrié C, Legeron R, Petit L et al (2018) Higher than standard dosing regimen are needed to achieve optimal antibiotic exposure in critically ill patients with augmented renal clearance receiving piperacillin-tazobactam administered by continuous infusion. J Crit Care 48:66-71. https:// doi.org/10.1016/j.jcrc.2018.08.026

10. Dhaese SAM, Thooft ADJ, Farkas A et al (2019) Early target attainment of continuous infusion piperacillin/tazobactam and meropenem in critically ill patients: a prospective observational study. J Crit Care 52:75-79. https://doi.org/10.1016/j.jcrc.2019.04.013

11. Abdul-Aziz MH, Alffenaar J-WC, Bassetti M et al (2020) Antimicrobial therapeutic drug monitoring in critically ill adult patients: a position paper. Intensive Care Med 46:1127-1153. https://doi.org/10.1007/ s00134-020-06050-1

12. van Lent-Evers NA, Mathôt RA, Geus WP et al (1999) Impact of goal-oriented and model-based clinical pharmacokinetic dosing of aminoglycosides on clinical outcome: a cost-effectiveness analysis. Ther Drug Monit 21:63-73. https://doi.org/10.1097/00007691-199902000-00010

13. Rybak MJ, Le J, Lodise TP et al (2020) Therapeutic monitoring of vancomycin for serious methicillin-resistant Staphylococcus aureus infections: a revised consensus guideline and review by the American Society of Health-system Pharmacists, the Infectious Diseases Society of America, the Pediatric Infectious Diseases Society, and the Society of Infectious Diseases Pharmacists. Clin Infect Dis 71:1361-1364. https://doi.org/10. 1093/cid/ciaa303

14. De Waele JJ, Carrette S, Carlier M et al (2014) Therapeutic drug monitoring-based dose optimisation of piperacillin and meropenem: a randomised controlled trial. Intensive Care Med 40:380-387. https://doi. org/10.1007/s00134-013-3187-2

15. Machado AS, Oliveira MS, Sanches C et al (2017) Clinical outcome and antimicrobial therapeutic drug monitoring for the treatment of infections in acute burn patients. Clin Ther 39:1649-1657.e3. https://doi.org/10. 1016/j.clinthera.2017.06.008

16. Richter DC, Frey O, Röhr A et al (2019) Therapeutic drug monitoringguided continuous infusion of piperacillin/tazobactam significantly improves pharmacokinetic target attainment in critically ill patients: a retrospective analysis of four years of clinical experience. Infection 47:1001-1011. https://doi.org/10.1007/s15010-019-01352-z

17. Roberts JA, Ulldemolins M, Roberts MS et al (2010) Therapeutic drug monitoring of beta-lactams in critically ill patients: proof of concept. Int J Antimicrob Agents 36:332-339. https://doi.org/10.1016/j.ijantimicag. 2010.06.008

18. Sime FB, Roberts MS, Tiong IS et al (2015) Can therapeutic drug monitoring optimize exposure to piperacillin in febrile neutropenic patients with haematological malignancies? A randomized controlled trial. J Antimicrob Chemother 70:2369-2375. https://doi.org/10.1093/jac/dkv123

19. Reinhart K, Brunkhorst FM, Bone H-G et al (2010) Prevention, diagnosis, therapy and follow-up care of sepsis: 1 st revision of S-2k guidelines of the German Sepsis Society (Deutsche Sepsis-Gesellschaft e.V. (DSG)) and the German Interdisciplinary Association of Intensive Care and Emergency Medicine (Deutsche Interdisziplinäre Vereinigung für Intensiv- und Notfallmedizin (DIVI)). Ger Med Sci 8:Doc14. https://doi.org/10.3205/000103

20. Hagel S, Fiedler S, Hohn A et al (2019) Therapeutic drug monitoringbased dose optimisation of piperacillin/tazobactam to improve outcome in patients with sepsis (TARGET): a prospective, multi-centre, randomised controlled trial. Trials 20:330. https://doi.org/10.1186/s13063-019-3437-x

21. Bergeron N, Dubois MJ, Dumont M et al (2001) Intensive Care Delirium Screening Checklist: evaluation of a new screening tool. Intensive Care Med 27:859-864. https://doi.org/10.1007/s001340100909

22. European Medicines Agency (2012) Reflection paper for laboratories that perform the analysis or evaluation of clinical trial samples, EMA/INS/ GCP/532137/2010

23. Moreno R, Vincent $J$, Matos R et al (1999) The use of maximum SOFA score to quantify organ dysfunction/failure in intensive care. Results of a prospective, multicentre study. Working Group on Sepsis related Problems of the ESICM. Intensive Care Med 25:686-696. https://doi.org/ 10.1007/s001340050931

24. Brunkhorst FM, Oppert M, Marx G et al (2012) Effect of empirical treatment with moxifloxacin and meropenem vs meropenem on sepsisrelated organ dysfunction in patients with severe sepsis: a randomized trial. JAMA 307:2390-2399. https://doi.org/10.1001/jama.2012.5833

25. Mouton JW, Muller AE, Canton R et al (2018) MIC-based dose adjustment: facts and fables. J Antimicrob Chemother 73:564-568. https://doi.org/10. 1093/jac/dkx427

26. Felton TW, Ogungbenro K, Boselli E et al (2018) Comparison of piperacillin exposure in the lungs of critically ill patients and healthy volunteers. J Antimicrob Chemother 73:1340-1347. https://doi.org/10.1093/jac/ dkx541 